\section{§5. Nonlinear Analysis for Stabilization of Interchange Mode in LHD Plasmas}

Ichiguchi, K., Nakajima, N., Carreras, B.A. (ORNL)

In the recent experiments in LHD, favorable MHD stability performance is observed in linearly unstable regime. To investigate the stabilizing mechanism in the LHD plasma, nonlinear MHD code NORM has been developed based on the reduced MHD equations ${ }^{1)}$. We apply the code to the LHD plasmas which is unstable against ideal interchange mode ${ }^{2)}$.

The nonlinear behavior of the interchange mode depends on the overlap of the vortices with different helicity. If the vortices are separated in the radial direction, the mode of each helicity saturates mildly with generating the local flat regions in the pressure profile. On the other hand, in the case of the significant overlap of the vortices, the vortices merges each other. The resultant large scale vortices cases a sudden global reductions.

Such significant overlap of the vortices can be suppressed by the continuous change of the pressure profile in the beta increase. This situation are realized numerically by employing a sequence of the saturated pressure profile in the increase of the beta value. Figure 1 shows the profile of average pressure at $\beta_{0}=0.5 \%, 1.0 \%$ and $1.5 \%$ in the sequence. These profile are obtained in the sequence of the saturated pressure profile from the 'Fixed' profile at $\beta_{0}=0.5 \%$. Interchange modes are mildly saturated at every beta value in this case.

Profiles in Fig. 1 show following two properties. One is in the local structure. Each pressure profile is locally flattened at the rational surfaces. These flat regions result from the exhaustion of the driving force by the resonant modes. Figure 2 shows the profiles of Mercier quantity $D_{I}$, which are calculated in equilibria with the saturated pressure profiles. The local stable regions are generated at the low order rational surfaces which corresspond to the local pressure flattening regions. The other one is in the global structure. The pressure profile is broadened gradually as the beta value is increased. .Figure 3 shows the comparison of the peaking factor with the experimental data ${ }^{3)}$. The tendency of the saturated pressure profile is quite consistent with the experimental results.

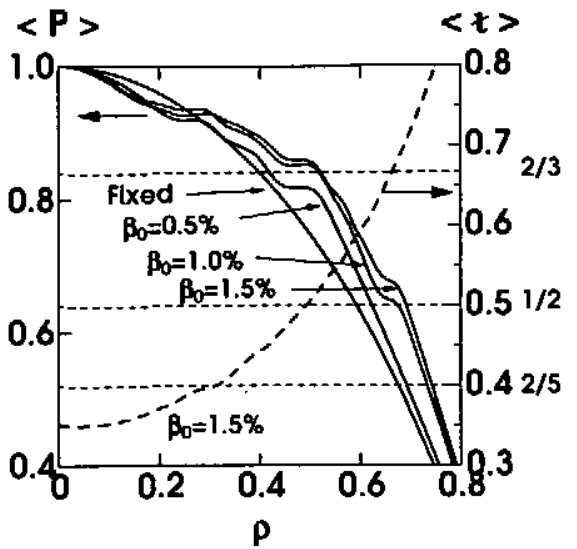

Fig.1 Saturated pressure profile.

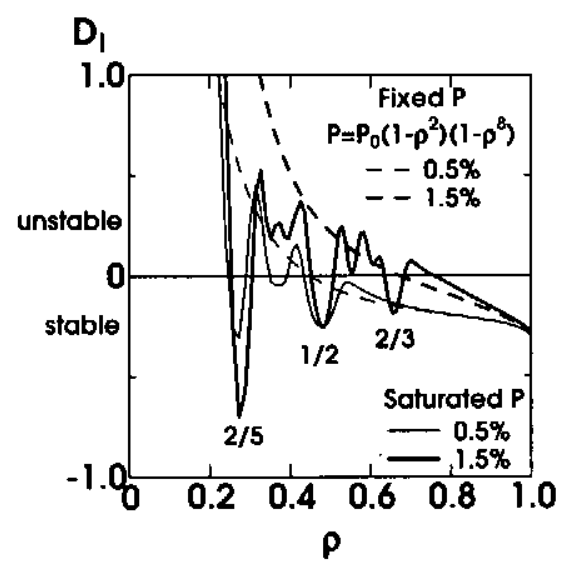

Fig.2 Profiles of Mercier quantity.

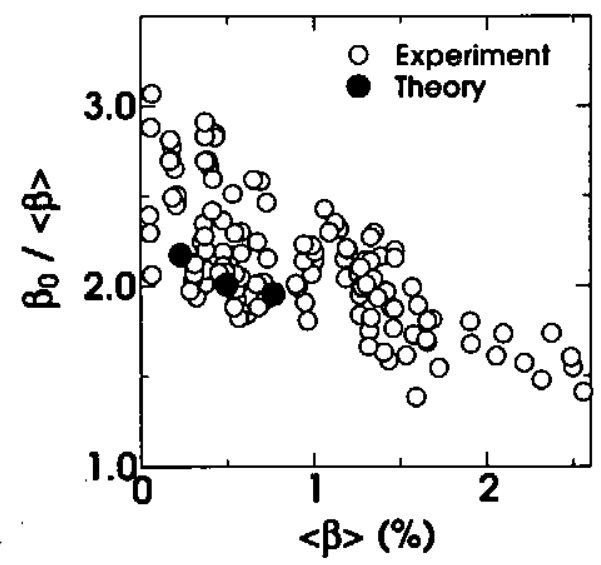

Fig.3 Peaking factor of pressure profile.

References

1) Ichiguchi, K., et al., Nuclear Fusion 43,(2003)1101.

2) Ichiguchi, K., et al., accepted in Fusion Science \& Technology (2004).

3) Sakakibara, S., et al., Plasma Phys. Contr. Fusion 44,(2002)A217. 